\title{
Contribution of energy systems during a Wingate power test
}

\author{
Jimmy C. Smith PhD and David W. Hill PhD \\ Department of Kinesiology, University of North Texas, Denton, Texas 76203-3857, USA
}

\begin{abstract}
Six men performed a total of 23 modified Wingate power tests against $5.5 \mathrm{kp}(53.9 \mathrm{~N})$ resistance on a Monark 864 ergometer. Breath-by-breath $\dot{V}_{2}$ was measured using a SensorMedics 4400 metabolic cart. Peak anaerobic power (highest $5 \mathrm{~s}$; mean(s.e.m.)) was 819(16) W (11.1(0.6) $\mathrm{W} \mathrm{kg}^{-1}$ ) and anaerobic capacity (work in $30 \mathrm{~s}$; mean(s.e.m.)) was $18.2(0.2) \mathrm{kJ}\left(248(11) \mathrm{J} \mathrm{kg}^{-1}\right)$. Contributions of ATP-PC, glycolytic and aerobic systems each $5 \mathrm{~s}$ were estimated. ATP-PC power (mean(s.e.m.)) peaked at 750(14) W $\left(10.2(0.6) \mathrm{W} \mathrm{kg}^{-1}\right.$ ) in the first $5 \mathrm{~s}$; glycolytic power (mean(s.e.m.)) peaked at $497(11) W\left(6.8(0.7) \mathrm{W} \mathrm{kg}^{-1}\right)$ between 10 and $15 \mathrm{~s}$ into the test; aerobic power (mean(s.e.m.)) peaked at 157(5) $\mathrm{W}\left(2.1(0.3) \mathrm{W} \mathrm{kg}^{-1}\right.$ ) during the last $5 \mathrm{~s}$ of the test, and $\dot{V}_{\mathrm{O}_{2}}$ exceeded $90 \% \dot{V}_{\mathrm{O}_{2} \text { peak }}$ Over the entire $30 \mathrm{~s}$, aerobic contribution was $16 \%$, glycolytic contribution was $56 \%$, and ATP-PC contribution was $28 \%$. It is concluded that glycolytic power peaks within the first $15 \mathrm{~s}$ of high power exercise; also, aerobic metabolism responds quickly during 'anaerobic' exercise and makes a significant contribution to the work performed.
\end{abstract}

Keywords: Anaerobic power, anaerobic capacity, work output, aerobic metabolism

The Wingate power test is used in the measurement of peak anaerobic power and anaerobic capacity (work in $30 \mathrm{~s}$ ). However, it is clear that even during short duration high power exercise, some ATP regeneration occurs through oxidative phosphorylation $^{1}$. The magnitude of the aerobic contribution to a 30-s high power exercise task has been estimated to be $9-19 \%^{2}, 28 \%^{3}$, or as high as $40 \%^{4}$ or $44 \%^{5}$.

In addition to calculating the aerobic contribution to exercise, it is possible to ascribe portions of the anaerobic component to either the ATP-PC or glycolytic systems. Any such estimation requires several assumptions, and previous studies have all made apparently different assumptions. Despite this limitation, it will be of value to have a relatively accurate assessment of the aerobic and anaerobic responses to short duration high power exercise.

The purpose of this study was to estimate the contributions of ATP-PC, glycolytic and aerobic

Address for correspondence: David W. Hill, Department of Kinesiology, PO Box 13857, University of North Texas, Denton, Texas 76203-3857, USA

(C) 1991 Butterworth-Heinemann Ltd 0306-3674/91/040196-04 systems to performance of measured work in a modified Wingate power test.

\section{Patients and methods}

A total of 23 modified Wingate power tests were performed on a Monark 864 ergometer (Varberg, Sweden) by six healthy male subjects. Subjects were of mean(s.d.) age 23(3) years, height $178(4) \mathrm{cm}$, mass $74.6(4.9) \mathrm{kg}$ and cycling $V_{\mathrm{O}_{2} \text {, peak }} 3138(294) \mathrm{ml} \mathrm{min}^{-1}$ (42.1(6.4) $\mathrm{ml} \mathrm{kg}^{-1} \mathrm{~min}^{-1}$ ). Each subject's tests were scheduled about 7 days apart. Resistance was $5.5 \mathrm{kp}$ $(53.9 \mathrm{~N})$ or mean(s.e.m.) $0.074(0.004) \mathrm{kp} \mathrm{kg}^{-1}$ $\left(0.73(0.04) \mathrm{N} \mathrm{kg}^{-1}\right)$ body mass.

Subjects were directed by tape-recorded instructions to begin slow unloaded pedalling. At $-10 \mathrm{~s}$, a countdown ('10, 9, 8, ..') was begun. All-out (still unloaded) pedalling began at $-4 \mathrm{~s}$ (' 4 ' on the countdown). Then, at $0 \mathrm{~s}$, the resistance was applied and the test began.

Peak power, or anaerobic power, the highest power produced in a 5-s segment of the test (always the first) was expressed in $W$ or $W \mathrm{~kg}^{-1}$. Anaerobic capacity, the total external work performed in the 30 -s test, was expressed in $\mathrm{kJ}$ or $\mathrm{J} \mathrm{kg}^{-1}$.

$\dot{V}_{\mathrm{O}_{2}}$ before and during the tests was determined on a breath-by-breath basis using a SensorMedics 4400 tc metabolic cart (Anaheim, California, USA). The $\mathrm{VO}_{2}$ data were averaged over 5 -s periods $(0-5 s, 6-10 s$, $11-15 \mathrm{~s}, 16-20 \mathrm{~s}, 21-25 \mathrm{~s}$ and $26-30 \mathrm{~s}$ ) to allow comparison of $\mathrm{VO}_{2}$ data with power and work data, which were collected at 5-s intervals.

Contribution of the various energy systems was calculated using methods and assumptions similar to those of Serresse and colleagues ${ }^{3}$.

ATP-PC contribution was calculated assuming $100 \%$ contribution until peak power was achieved, and assuming that peak power was achieved at the 2.5-s point in the test. It has been estimated that less than $1 \mathrm{~s}$ may be required to reach peak ATP turnover from ATP-PC stores ${ }^{6}$. Sargeant and Dolan have reported that peak power occurs $1-4 \mathrm{~s}$ into exercise that is performed under conditions similar to those in the present study ${ }^{7}$; the 2.5-s value selected by Serresse and colleagues, ${ }^{3}$, and used in the present study, is midway between these points. Then, ATP-PC contribution was assumed to decline linearly until the 10-s 
point. A postulated linear decline in ATP-PC contribution to the 10-s point, also used by Serresse and colleagues $^{3}$, represents a somewhat arbitrary assumption. It is known that ATP-PC stores are the primary source early in exercise, as they show an almost immediate increase to peak ATP turnover ${ }^{6}$, whereas glycolysis requires about $5 \mathrm{~s}^{6}$; aerobic metabolism, while initially limited by peripheral metabolic adjustments ${ }^{8}$, responds with a half time of less than $30 \mathrm{~s}$. Thus, calculation of the ATP-PC contribution was based solely on the work performed in the first $5 \mathrm{~s}$ of the test.

Aerobic contribution was calculated assuming a linear increase from 0 at $2.5 \mathrm{~s}$ until the 10-15-s portion of the test; $\mathrm{VO}_{2}$ kinetics (when cycling exercise is preceded by unloaded pedalling, as in this protocol) are described by a monoexponential function with a half time of less than $30 \mathrm{~s}$; the initial $10 \mathrm{~s}$ of such a fast-rising response is essentially linear ${ }^{10}$. For the $10-15-s$ portion and for subsequent 5-s intervals, aerobic contribution was calculated from the measured $\dot{\mathrm{V}}_{2}$ using $20.92 \mathrm{kJI}^{-1} \mathrm{O}_{2}$ assuming $100 \%$ carbohydrate use, and adjusted for efficiency of $18.5 \%$ (i.e. after subtraction of measured baseline $\left.\mathrm{V}_{2}\right)$; an efficiency value of $18.5 \%$ was selected, based on the high power outputs and pedalling frequencies associated with this type of test.

The glycolytic contribution was determined by subtraction of the ATP-PC and aerobic contributions from the total work performed.

\section{Results}

Peak anaerobic power (mean(s.e.m.)) was 819(16) W (11.1(0.6) $\mathrm{W} \mathrm{kg}^{-1}$ ), and anaerobic capacity (mean(s.e.m.)) was 18.2(0.2) kJ (248(11) $\mathrm{J} \mathrm{kg}^{-1}$ ).

ATP-PC contribution data are presented in Figure 1. ATP-PC power (over a 5-s period; mean(s.e.m.)) peaked at 750(14) W (10.2(0.6) $\left.\mathrm{W} \mathrm{kg}^{-1}\right)$, and ATP-PC capacity (mean(s.e.m.)) was $5.1(0.5) \mathrm{kJ}\left(69(4) \mathrm{kJ} \mathrm{kg}^{-1}\right.$ ). There was (assumed) a rapid decline in the ATP-PC contribution from its peak to the 10 -s point.

Glycolytic contribution data are presented in Figure 2. Glycolytic contribution (mean(s.e.m.)) increased until the 10-15-s interval, when the contribution was the maximal power of the glycolytic system, 497(11) W (6.8(0.7) $\left.\mathrm{W} \mathrm{kg}^{-1}\right)$. Total work performed through glycolysis during the 30 -s Wingate test was $10.2(0.3) \mathrm{kJ}$ (138)(9) $\left.\mathrm{J} \mathrm{kg}^{-1}\right)$.

Aerobic contribution data are presented in Figure 3. Aerobic contribution gradually increased throughout the test. During the last 5-s segment of the test, $\dot{V O}_{2}$ exceeded $90 \%$ of $\dot{V}_{\mathrm{O}_{2} \text { peak }}$ and aerobic mechanisms provided $35 \%$ of the energy necessary for ATP regeneration.

Total power production each $5 \mathrm{~s}$ declined from $819(16) \mathrm{W}$ in the first $5 \mathrm{~s}$ to $450(10) \mathrm{W}$ in the final $5 \mathrm{~s}$. This is a reduction of $45 \%$. Over the course of the 30 -s test, $56 \%$ of the energy was from glycolysis, $28 \%$ was from ATP-PC stores, and $16 \%$ was from aerobic sources. The contributions of the energy systems during the time of peak output for each system are presented in Table 1 .

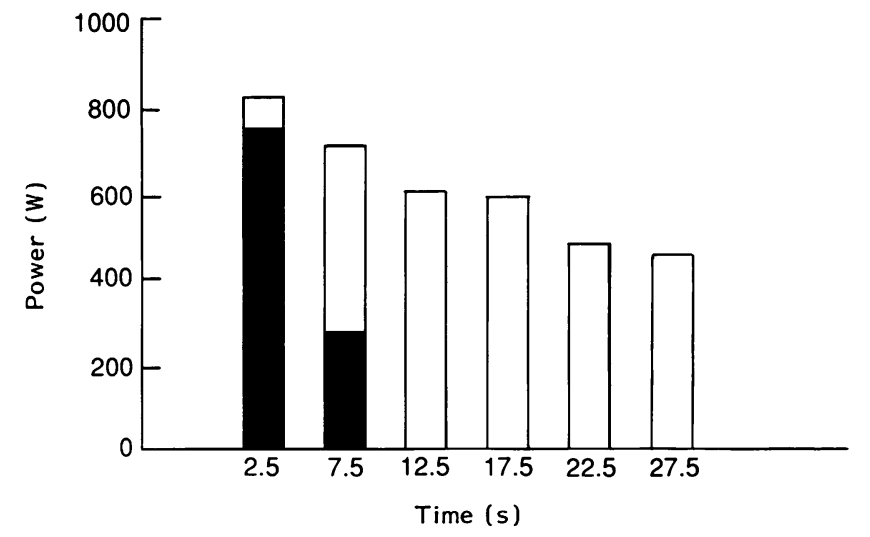

Figure 1. ATP-PC contribution (W) at each 5-s interval. ATP-PC contributions are superimposed on total power output in each 5-s interval

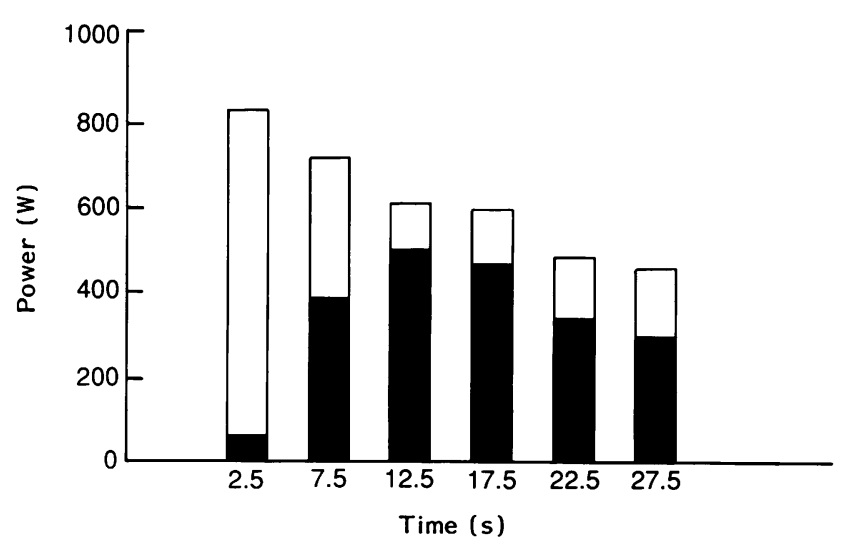

Figure 2. Glycolytic contribution (W) at each 5-s interval. Glycolytic contributions are superimposed on total power output in each 5-s interval

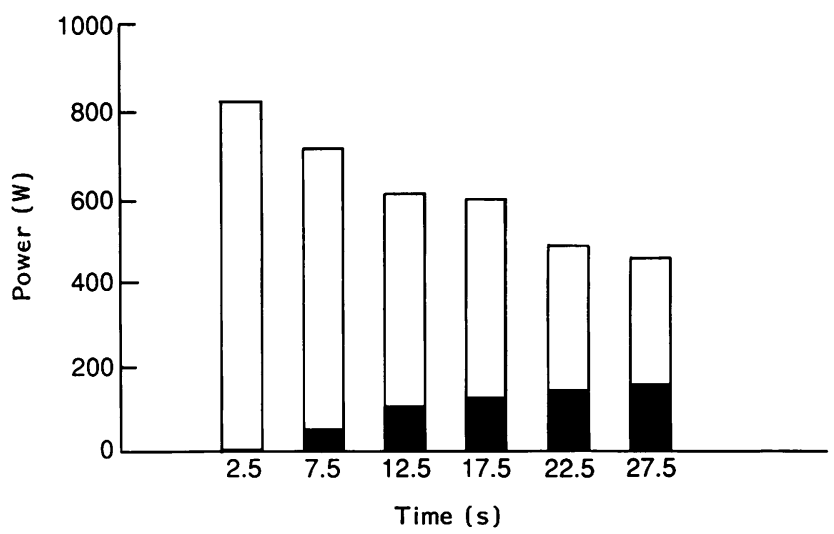

Figure 3. Aerobic contribution (W) at each 5-s interval. Aerobic contributions are superimposed on total power output in each 5-s interval

Table 1. Total power produced (i.e. 'peak power' or 'peak anaerobic power'), and peak power of the various energy systems

\begin{tabular}{llcc}
\hline Energy system & $W$ & $W k^{-1}$ & Time (s) \\
\hline Peak power & $819(16)$ & $11.1(0.6)$ & First 5 \\
ATP-PC power & $750(14)$ & $10.2(0.6)$ & First 5 \\
Glycolytic power & $497(11)$ & $6.7(0.7)$ & $10-15$ \\
Aerobic power & $157(5)$ & $2.1(0.3)$ & $25-30$ \\
\hline
\end{tabular}

Values are means (s.e.m.) 


\section{Discussion}

\section{Anaerobic power and capacity}

Peak anaerobic power (mean(s.e.m.)) was 819(16) W (11.1(0.6) $\mathrm{W} \mathrm{kg}^{-1}$ ), and anaerobic capacity (mean (s.e.m.)) was $18.2(0.2) \mathrm{kJ}\left(248(11) \mathrm{J} \mathrm{kg}^{-1}\right)$. These are similar to the values of Serresse and colleagues ${ }^{3}$ who reported means of $9.8 \mathrm{~W} \mathrm{~kg}^{-1}$ and $260 \mathrm{~J} \mathrm{~kg}^{-1}$, respectively, in 21 men. Expressed as the mean power over $30 \mathrm{~s}$, the anaerobic capacity was $8.3(0.4) \mathrm{W} \mathrm{kg}^{-1}$, similar to that reported in other studies ${ }^{10}$.

$\mathrm{V}_{\mathrm{O}_{2}}$ during the final 5-s segment was $93 \% \dot{\mathrm{V}}_{2}$ peak demonstrating that aerobic metabolism responds quickly to the energy demands of high-intensity exercise.

\section{ATP-PC power}

ATP-PC power (in the first 5-s of the test; mean(s.e.m.)) was 750(14) W (10.2(0.6) $\mathrm{W} \mathrm{kg}^{-1}$ ), which (understandably) is lower than the $840 \mathrm{~W}$ $\left(12.0 \mathrm{~W} \mathrm{~kg}^{-1}\right)$ estimated by Serresse and colleagues ${ }^{3}$ for a 1-s segment in a 10-s test. The ATP-PC capacity (mean(s.e.m.)) was $5.1(0.5) \mathrm{kJ}^{\left(69(4) \mathrm{J} \mathrm{kg}^{-1}\right.}$ ), which is similar to the $4 \mathrm{~kJ}\left(60 \mathrm{~J} \mathrm{~kg}^{-1}\right)$ estimated by Serresse and colleagues ${ }^{3}$.

\section{Glycolytic power}

Glycolytic power (mean(s.e.m.)) peaked at 497(11) W $\left(6.8(0.7) \mathrm{W} \mathrm{kg}^{-1}\right)$ between 10 and $15 \mathrm{~s}$ after the beginning of a 30-s Wingate test. Serresse and colleagues $^{3}$ used 15-s samples and concluded that the maximal glycolytic power was achieved $15-30 \mathrm{~s}$ into a 90-s effort, against the same relative resistance that we used. They concluded that glycolytic power would peak at the same time in a shorter (i.e. 30-s) test. Like Serresse and colleagues, we can report higher glycolytic contribution during the second $15 \mathrm{~s}$ of the test than the first $15 \mathrm{~s}$. However, we were able to evaluate the progress of contribution from the various energy systems using smaller time frames (5-s increments) and we report that maximal power of the glycolytic system actually occurs near the end of the first $15 \mathrm{~s}$, about 10-15s after the beginning of all-out loaded pedalling in a 30-s Wingate power test. This demonstrates that glycolytic power (as opposed to anaerobic power) may be estimated from a 20 -s or even a 15-s test. Total work performed through glycolysis (mean (s.e.m.)) was 10.2(0.3) kJ (138(9) $\left.\mathrm{J} \mathrm{kg}^{-1}\right)$, which is understandably lower than the value of $210 \mathrm{~J} \mathrm{~kg}^{-1}$ reported for work completed in a $90-\mathrm{s}$ effort in the study of Serresse and colleagues ${ }^{3}$.

\section{Aerobic contribution}

There are two approaches to the problem of estimating aerobic contribution. Stevens and colleagues ${ }^{5}$, Kavanagh and Jacobs ${ }^{2}$, Serresse and colleagues $^{3}$, and this study essentially measured oxygen uptake, used the resulting $\mathrm{VO}_{2}$ to estimate aerobic contribution, and then estimated anaerobic contribution based on the difference between total work completed and estimated aerobic work that was performed. Medbø and Tabata $^{4}$ measured total power output, used this to estimate the oxygen cost of all work completed, and then determined anaerobic contribution as the difference between the estimated oxygen cost and the measured oxygen uptake (i.e. oxygen deficit). The first method assumes an efficiency for aerobic metabolism to estimate aerobic power, and the second method assumes an efficiency for aerobic plus anaerobic metabolism to estimate total oxygen cost. The different assumptions of the two methods may contribute to the differences between values reported for the relative importance of aerobic and anaerobic processes in short-term all-out exercise.

Our values for aerobic contribution are much lower than the $44 \%$ suggested by Stevens and colleagues ${ }^{5}$. They reported data from 13 tests and assumed that $\mathrm{V}_{\mathrm{O}_{2}}$ measured at the mouth reflected tissue $\dot{V}_{2}$ and that there was no cardiodynamic effect or lag between occurrence of an event at the tissues and appearance of changes (i.e. in venous $\mathrm{O}_{2}$ content) at the lung. They may have overestimated aerobic metabolism during the initial stages of the tests.

Our values are also lower than the $40 \%$ estimated by Medbø and Tabata ${ }^{4}$. They evaluated 14 subjects exercising on a cycle ergometer at a work rate which resulted in exhaustion (mean(s.e.m.)) at 34(2) s, a task similar to a Wingate test. Their protocol differed from a Wingate in two ways. First, duration was longer and it would therefore be expected that overall aerobic contribution would be higher than in a 30-s test. Second, power output (mean(s.e.m.)) was constant $\left(9.1(0.2) \mathrm{W} \mathrm{kg}^{-1}\right)$ and higher than the mean 30 -s power output in this study $\left(8.3(0.4) \mathrm{W} \mathrm{kg}^{-1}\right)$. Their estimates of exercise $\dot{V}_{2}$ were based on single 30-s Douglas bag samples. They calculated aerobic contribution based on total $\mathrm{V}_{2}$ (i.e. assuming that all measured $\mathrm{VO}_{2}$ was used in energy production) and added an extra c. $30 \%$ to account for the use of stored oxygen during the initial stages of the test.

Our values are also lower than the $28 \%$ of Serresse and colleagues ${ }^{3}$. These investigators analysed data from 21 tests of fit individuals, and based their estimates on a single 30-s Douglas bag sample. Thus, although they concluded that the aerobic contribution did not begin at the onset of activity, like Stevens and colleagues, they based their calculations on the premise that all oxygen consumed during the 30-s was contributing to the external work. This might explain the difference between their estimated $28 \%$ aerobic contribution and our estimated $16 \%$ contribution.

Kavanagh and Jacobs $^{2}$ used breath-by-breath analyses of expired air and reported an aerobic contribution of $19 \%$, based on data from five tests. Their value for efficiency was $25 \%$, with subtraction of a 1 MET (metabolic equivalent, $3.5 \mathrm{ml} \mathrm{kg}^{-1} \mathrm{~min}^{-1}$ ) baseline $\mathrm{VO}_{2}$. They also performed calculations with efficiencies of $20 \%$ and $13 \%$, and reported aerobic contributions would then be calculated to be $14 \%$ or $9 \%$.

We used an efficiency of $18.5 \%$, after subtracting measured baseline $\hat{V O}_{2}$ (almost two METs) from exercise $\mathrm{VO}_{2}$. Our estimate of $16 \%$ aerobic contribu- 
tion is similar to that of Kavanagh and Jacobs ${ }^{2}$, and suggests that oxidative phosphorylation makes a significant contribution to meeting the energy demands in tests of 'anaerobic' capacity.

\section{Selection of the efficiency value}

Selection of the $18.5 \%$ value for net efficiency used in this study was based on the high power outputs and pedal frequencies encountered in this kind of testing. Any value used is only a 'best guess', since efficiency or economy can be measured only during steady state exercise. Others have used different values, such as $16.2 \%$ (with no baseline subtraction) which was reported to be the subjects' mean value achieved at the end of a $\dot{V}_{2}$, max test, although it is not clear how they corrected for the anaerobic contribution during this test ${ }^{3}$. Kavanagh and Jacobs ${ }^{2}$ calculated aerobic contributions using the $25 \%$ net efficiency proposed by Gaesser and Brooks ${ }^{11}$. Medbø and Tabata ${ }^{4}$ measured efficiency during repeated 10-min exercise bouts at various power outputs, demanding from $30 \%$ to $90 \%$ of $\mathrm{V}_{2 \max }$ reported a linear increase in $\dot{V}_{2}$ with work rate, and assumed that the linearity would hold as work rate increased from $90 \%$ of $\dot{V}_{\mathrm{O}_{2} \text { max }}$ to about $185 \%$ of the $\dot{V} \mathrm{O}_{2}$ max demands of the 34-s exercise bout.

Recently, Davies and Sandstrom ${ }^{12}$ have suggested that aerobic and anaerobic processes share a common gross efficiency of $22 \%$. It is our contention that this value might overestimate the aerobic contribution to measured work in a high power output task such as the Wingate test because: (1) high velocity of movement (c.150 r.p.m. in the first $5 \mathrm{~s}$ ) would be expected to be less efficient; and (2) upper body muscles were exerting a considerable amount of (unmeasured) force against the handlebars. Nevertheless, we calculated power of the ATP-PC, glycolytic and aerobic systems, and overall aerobic contribution, using $25 \%$ net and $22 \%$ gross efficiencies. Results are presented in Table 2. A net efficiency of $25 \%$ suggested by Gaesser and Brooks ${ }^{11}$ yielded $22 \%$ overall aerobic contribution; the gross efficiency of $22 \%$ proposed by Davies and Sandstrom ${ }^{12}$ yielded $24 \%$ overall aerobic contribution. We feel that the range of values, $16-24 \%$, calculated using these different and defensible values for efficiency, accurately represents the significant aerobic contribution during a 30-s Wingate test.

Table 2. Comparison of contributions of various energy systems when calculations were made with different choices for efficiency: $18.5 \%$ net efficiency ( $18.5 \%$ net) versus $25 \%$ net efficiency ( $25 \%$ net) versus $22 \%$ gross efficiency ( $22 \%$ gross). Contributions are given as power outputs $(W)$ and as percentages of the total power produced in the 5-s interval

\begin{tabular}{llll}
\hline Energy system & $18.5 \%$ net & $25 \%$ net & $22 \%$ gross \\
\hline ATP-PC power & $750(14)(92 \%)$ & $750(14)(92 \%)$ & $750(14)(92 \%)$ \\
Glycolytic power & $497(11)(82 \%)$ & $460(12)(76 \%)$ & $438(12)(72 \%)$ \\
Aerobic power & $157(5)(35 \%)$ & $212(7)(47 \%)$ & $225(5)(51 \%)$
\end{tabular}

\section{Summary of findings}

Our results suggest that a single 30 -s test can be used to estimate ATP-PC power and capacity - these two values are based solely on application of assumptions and measurement of peak power. Such a test can also be used to estimate peak glycolytic power. Moreover, a traditional indicator of anaerobic capacity, work performed in $30 \mathrm{~s}$, may be obtained; glycolytic capacity may be estimated by subtraction. However, it is clear that a 30-s test is too short to quantify glycolytic or anaerobic capacity, and anaerobic and glycolytic capacities will be underestimated by such a short test.

Over the 30-s course of the Wingate power test, $16 \%$ (or perhaps as much as $24 \%$ ) of the total ATP regeneration is via aerobic mechanisms and, during the final $5 \mathrm{~s}$ of the test, aerobic contribution is $35 \%$. It is concluded that glycolytic power peaks within the first $15 \mathrm{~s}$ of all-out exercise; also, aerobic metabolism responds quickly to demands of 'anaerobic' exercise and makes a significant contribution to the work performed.

\section{Acknowledgements}

This research was supported by a University of North Texas (UNT) Faculty Research Grant.

\section{References}

1 Vandewalle $H$, Pérès $G$, Monod $H$. Standard anaerobic exercise tests. Sports Med 1987; 4: 268-89.

2 Kavanagh MF, Jacobs I. Breath-by-breath oxygen consumption during performance of the Wingate Test. Can J Sport Sci 1988; 13: 91-3.

3 Serresse O, Lortie G, Bouchard C, Boulay MR. Estimation of the contribution of the various energy systems during maximal work of short duration. Int J Sports Med 1988; 9: 456-60.

4 Medbø JI, Tabata I. Relative importance of aerobic and anaerobic energy release during short-lasting exhausting bicycle exercise. J Appl Physiol 1989; 67: 1881-6.

5 Stevens GHJ, Wilson BW, Raven PB. Aerobic contribution to the Wingate test. Med Sci Sports Exerc 1986; 18 (Suppl): 2.

6 Lewis SF, Haller RG. Skeletal muscle disorders and associated factors that limit exercise performance. In: Pandolf KB, ed. Exer Sport Sci Rev 1989: 67-113.

7 Sargeant AJ, Dolan P. Effect of prior exercise on maximal short-term power output in humans. J Appl Physiol 1987; 63: 1475-80.

8 Sahlin K, Ren JM, Brobert S. Oxygen deficit at the onset of submaximal exercise is not due to delayed oxygen transport. Acta Physiol Scand 1988; 134: 175-80.

9 DIPrampero PE, Mahler PB, Giezendanner D, Cerretelli P. Effects of priming exercise on $\mathrm{VO}_{2}$ kinetics and $\mathrm{O}_{2}$ deficit at the onset of stepping and cycling. J Appl Physiol 1989; 66: 2023-31.

10 Maud PJ, Shultz BB. Norms for the Wingate Anaerobic Test with comparison to another similar test. Res Q Exer Sport 1989; 60: 144-51.

11 Gaesser GA, Brooks GA. Muscular efficiency during steadyrate exercise: effect of speed and work rate. I Appl Physiol 1975; 38: 1132-9.

12 Davies CTM, Sandstrom ER. Maximal mechanical power output and capacity of cyclists and young adults. Eur J Appl Physiol 1989; 58: 838-44. 